\title{
Controlling the Inspiration/Expiration Ratio Benefits the Deceleration Capacity Index of Heart Rate in Assessing the Sympatho-vagal Balance
}

\author{
Qing Pan ${ }^{1}$, Chenglong Gao ${ }^{1}$, Gongzhan Zhou ${ }^{1}$, Ruofan Wang ${ }^{2}$, \\ Yihua $\mathrm{Yu}^{3}$, Luping Fang ${ }^{1}$, Gangmin Ning $^{2}$ \\ ${ }^{1}$ College of Information Engineering, Zhejiang University of Technology, Hangzhou, China \\ ${ }^{2}$ Department of Biomedical Engineering, Zhejiang University, Hangzhou, China \\ ${ }^{3}$ Department of ICU, Zhejiang Hospital, Hangzhou, China
}

\begin{abstract}
Deceleration capacity (DC) of heart rate is a novel index for evaluating the activity of the autonomic nervous system (ANS). We examined whether controlling the inspiration/expiration (I/E) ratio benefits the DC analysis based on a model-generated RR interval (RRI) database. A cardiovascular system model was adopted to simulate RRI time series. The model allows analyzing the role of sympathetic and vagal activities in the ANS. The respiratory pattern can be controlled in the model.

Three hundred RRI time series with random sympathetic and vagal activities were simulated. According to the ratio between the sympathetic and vagal activities ( $S / V$ ratio), these subjects were categorized into a case group $(S / V>1)$ and a control group $(S / V<1)$. $D C$ was computed for each subject. The performance of $D C$ in distinguishing the two groups was examined by the receiver operating characteristic (ROC) analysis. The respiratory period is set to $6 \mathrm{~s}$. The I/E ratio was controlled as 1:2, 1:1 and 2:1, respectively, and the performances of $D C$ under different $I / E$ ratios were compared.

The numbers of subjects in the case group and the control group are 139 and 161 . With the I/E ratio as 1:2, $1: 1$, and $2: 1$, the mean area under the ROC curves (AUCs) of DC are 0.64, 0.74 and 0.75. DCs obtained with the I/E ratio of 1:1 and 2:1 have significantly larger AUCs than that obtained under the normal physiological I/E ratio of $1: 2(p<0.05)$.

Controlling the I/E ratio above the normal physiological level renders a better ability of $D C$ in assessing the sympatho-vagal balance.
\end{abstract}

\section{Introduction}

Deceleration capacity (DC) and acceleration capacity (AC) of heart rate are a pair of novel indices for the evaluation of autonomic nervous system (ANS) [1]. They are obtained by applying a phase-rectified signal averaging (PRSA) algorithm to the RR interval (RRI) series [2]. The PRSA selects the decelerating or the accelerating RRIs as the anchor points. The sections around the anchor points are defined and averaged to produce an averaging RRI series. DC or AC is computed as the coefficient of the Haar wavelet at scale two in the center of the averaging RRI series.

The performances of DC and AC are distinct [1, 3, 4]. Based on a cardiovascular system model, we recently demonstrated that the degree of heart rate asymmetry (HRA) influences the performance of DC and AC in assessing the activity of ANS [5]. It implies that the performance of DC can be improved by adjusting the HRA level. As the inspiration/expiration (I/E) ratio influences the HRA level [6, 7], we attempted to analyze whether controlling the I/E ratio benefits DC in assessing the ANS activity.

Our study was conducted based on a cardiovascular system model. The model can produce a set of RRI time series with controlled levels of ANS activity for DC calculation. It can also control the $\mathrm{I} / \mathrm{E}$ ratio to examine its role in the performance of DC.

\section{Methods}

The study was conducted in three steps. First, we developed the cardiovascular system model to simulate the RRI time series. Second, we generated a set of RRI time series with random ANS activities and controlled respiratory $\mathrm{I} / \mathrm{E}$ ratios as the virtual subjects for the study. Third, we computed DC for all the subjects and analyzed the performance of DC under different respiratory patterns.

\subsection{Model development}

The block diagram of the cardiovascular system model is shown in Figure 1. Details of the model can be referred in the literature $[8,9]$. It contains a hemodynamic model 
and an ANS model. The hemodynamic model includes the heart, the systemic circulation and the pulmonary circulation. Each chamber of the heart or the vessel is represented as a Windkessel compartment with a resistant element and a compliant element. The systemic and pulmonary arteries also involve an inertial element. The effect of respiration is applied by setting the breathdependent extravascular pressures in the intrathoracic and abdominal cavities.

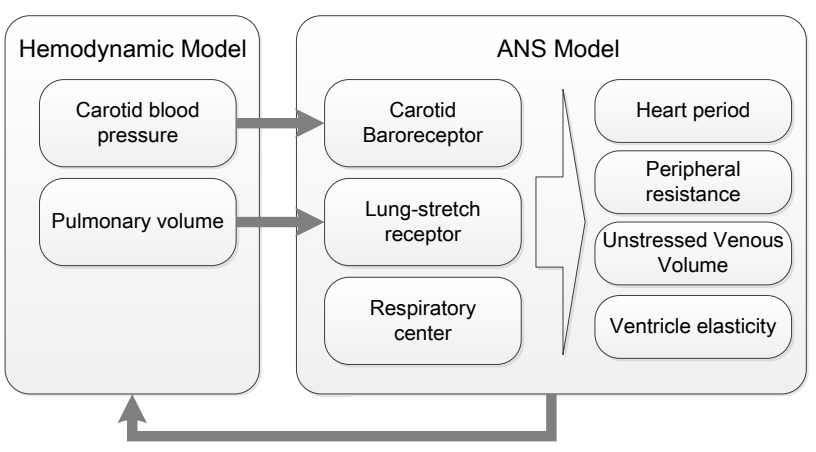

Figure 1. Block diagram of the cardiovascular system model

The ANS model regulates the heart period, the peripheral resistance, the unstressed venous volume, and the ventricle elasticity. The heart period is mainly regulated by the joint effects of the sympathetic and vagal activities through their responses to the stimuli from the carotid baroreceptors and the lung-stretch receptors. The role of respiratory center is also considered.

Since DC is a variant of HRV index, we verified the model in simulating heart rate variability (HRV). In particular, the ratio between the low-frequency (LF) component and the high-frequency (HF) component in the power spectral density of HRV was computed for the validation. The frequency range is 0.04 to $0.15 \mathrm{~Hz}$ for the LF component and 0.15 to $0.4 \mathrm{~Hz}$ for the HF component. The ability of the model in simulating the HRA phenomenon was validated by checking whether the relationship between the HRA and the I/E ratio agrees with the experimental findings [6, 7].

\subsection{Simulation protocol}

All the model parameters were set according to the literature $[8,9]$. The respiratory period was set to $6 \mathrm{~s}$. The normal I/E ratio (1:2), and two higher I/E ratios (1:1 and $2: 1$ ) were set in the model respectively. Under each $\mathrm{I} / \mathrm{E}$ ratio, three hundred RRI time series were simulated as virtual subjects. In each simulation, the sympathetic and vagal activities, and the time delay and the time constant of the ANS were randomly set within $\pm 20 \%$ of their basal values. According to the ratio between the sympathetic and vagal activities ( $\mathrm{S} / \mathrm{V}$ ratio), these subjects were categorized into a case group $(\mathrm{S} / \mathrm{V}>1)$ and a control group $(\mathrm{S} / \mathrm{V}<1)$ in consideration of the fact that many cardiovascular diseases (CVDs) exhibit sympathetic overactivity [10].

\subsection{Data analysis}

DC was computed by applying the PRSA algorithm to the simulated RRI time series [2]. In brief, the $i$ th $R R I$ was defined as the anchor point if it satisfies

$$
R R I_{i}>R R I_{i-1}
$$

A section with length of $2 L+1$ was defined around each anchor point. $L$ was defined as 60 . All the sections were aligned according to the anchor points and averaged. The average was represented as $\overline{R R I}$. DC was calculated as

$$
\mathrm{DC}=[\overline{R R I}(0)+\overline{R R I}(1)-\overline{R R I}(-1)-\overline{R R I}(-2)] / 4
$$

where the index 0 indicates the position of the anchor point.

The Porta Index (PI) and Guzik Index (GI) were used to quantify the degree of HRA [11]. PI quantifies the uneven distribution of the positive and negative RRI variations. Given the series of beat-to-beat RRI variation as

$$
\Delta R R I=\left\{\Delta R R I_{i}=R R I_{i+1}-R R I_{i}, i=1, \ldots, N-1\right\}
$$

PI was calculated as

$$
P I=\frac{N\left(\Delta R R I^{-}\right)}{N(\Delta R R I \neq 0)} \times 100 \%
$$

where $N\left(\Delta R R I^{-}\right)$is the number of negative RRI variations and $N(\triangle R R I \neq 0)$ is the number of non-zero RRI variations. GI was calculated as

$$
G I=\frac{\sum\left(\Delta R R I_{i}^{+}\right)^{2}}{\sum\left(\Delta R R I_{i}\right)^{2}} \times 100 \%
$$

Each simulation ran for $1200 \mathrm{~s}$ and the data of the last $1000 \mathrm{~s}$ were used for the calculation of DC and HRA indices. A student t-test was used to analyze the difference between the case and the control groups. The receiver operating characteristic (ROC) curve was adopted to evaluate the performance of DC under different I/E ratios.

\section{Results}

\subsection{Validity of the model}

The LF/HF ratio of HRV is shown in Figure 2. The ratio is 0.82 at the basal condition. When the sympathetic activity was modified by $\pm 20 \%$, the $\mathrm{LF} / \mathrm{HF}$ ratios were obtained as 1.59 and 0.48 , respectively. When the vagal activity was modified by $\pm 20 \%$, the $\mathrm{LF} / \mathrm{HF}$ ratios were 
obtained as 0.40 and 1.56 , respectively. It agrees with the general understanding that the LF/HF ratio reflects the sympatho-vagal balance.

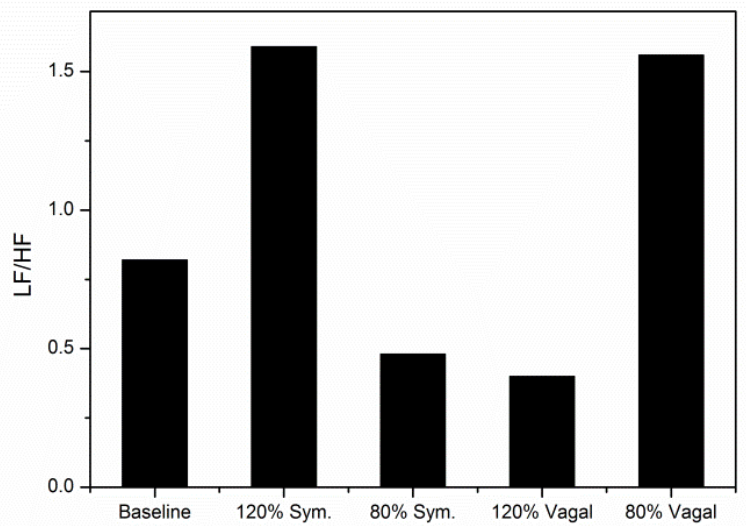

Figure 2 LF/HF ratio under different ANS activities. Sym.: sympathetic activity. Vagal: vagal activity

Figure 3 shows that the HRA increases monotonously with the elevation of the I/E ratio. When the $\mathrm{I} / \mathrm{E}$ ratio was modified from 1:2 to 2:1, PI increases from $42.7 \pm 2.7 \%$ to $63.7 \pm 2.3 \%$, and GI increases from $45.3 \pm 2.1 \%$ to $61.5 \pm 2.0 \%$, in agreement with the reported physiological experiments [6, 7].
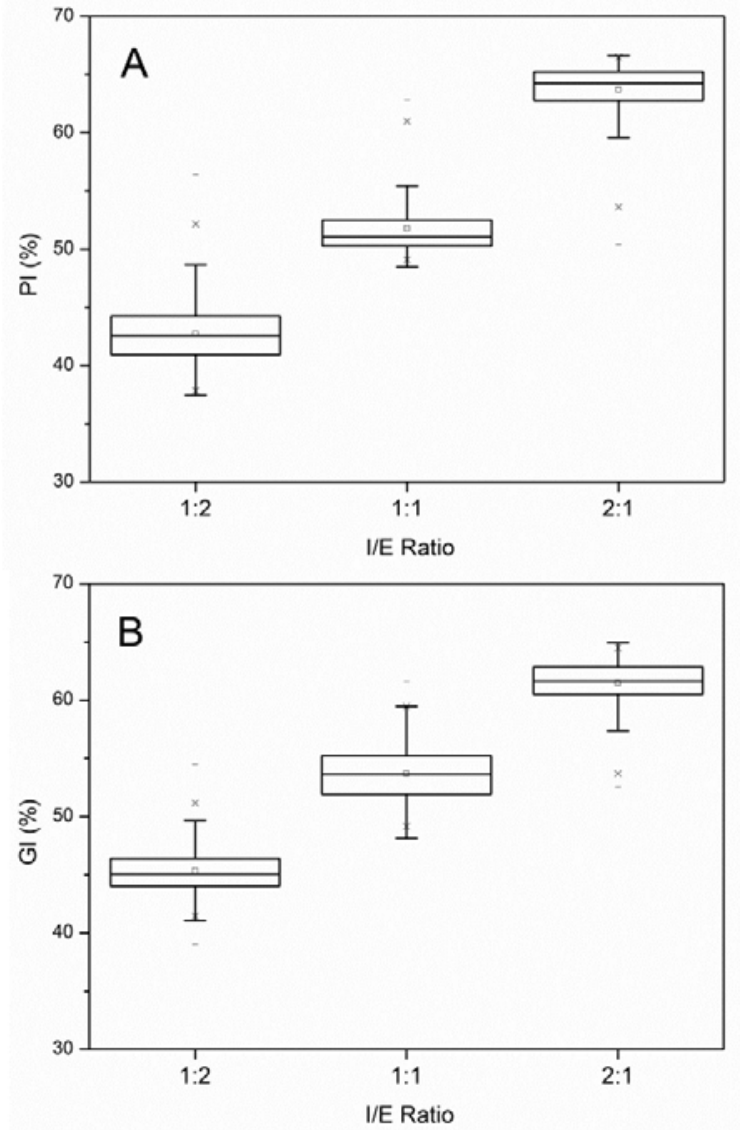

Figure 3 Relationship between the HRA indices and the I/E ratio. (A) PI vs. I/E ratio. (B) GI vs. I/E ratio

\subsection{Performance of DC}

The numbers of subjects in the case group and the control group are 139 and 161, respectively. The computed DCs under the I/E ratios of 1:2, 1:1 and 2:1 are given in Table 1 . It is observed that DC is significantly higher in the control group under all the $\mathrm{I} / \mathrm{E}$ ratios, supporting that DC is a vagal index. It is also observed that the DC values are higher when the subjects breathe in a higher I/E ratio. It suggests that breathing with a higher I/E ratio can better extract the quasi-periodic components in the RRI time series.

Table 1 DC values under different I/E ratios for the case and the control groups

\begin{tabular}{lcccc}
\hline \multirow{2}{*}{ Group } & \multirow{2}{*}{ Size } & \multicolumn{3}{c}{$\mathrm{DC}(\mathrm{ms})$} \\
\cline { 3 - 5 } & $\mathrm{I} / \mathrm{E}=1: 2^{*}$ & $\mathrm{I} / \mathrm{E}=1: 1^{*}$ & $\mathrm{I} / \mathrm{E}=2: 1^{*}$ \\
\hline Control & 161 & $19.3 \pm 3.2$ & $24.9 \pm 3.7$ & $30.3 \pm 5.1$ \\
Case & 139 & $17.5 \pm 3.5$ & $21.5 \pm 4.2$ & $25.7 \pm 5.3$ \\
\hline *: $\mathrm{p}<0.001$ & & &
\end{tabular}

The ROC curves for the three I/E ratios are shown in Figure 4 . The area under the ROC curves (AUCs) are $0.64,0.74$, and 0.75 for the $\mathrm{I} / \mathrm{E}$ ratio of $1: 2,1: 1$, and 2:1, respectively. The AUCs for I/E ratio of $1: 1$ and $1: 2$ are significantly larger than that for the I/E ratio of $1: 2(\mathrm{p}<$ 0.05). It indicates that the DCs obtained with an I/E ratio higher than the normal physiological value exhibit a significantly better performance in assessing the sympatho-vagal balance.

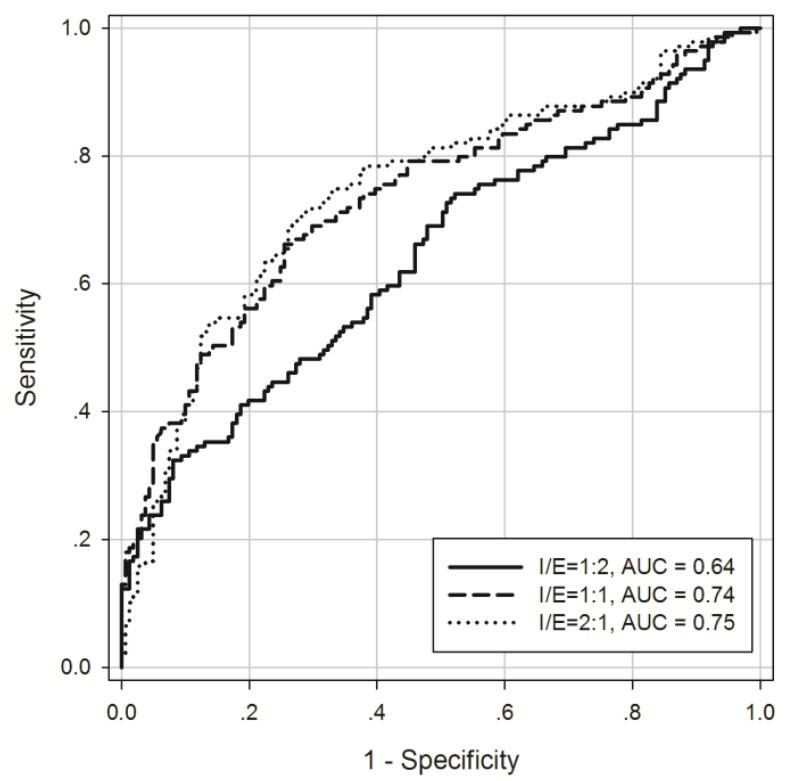

Figure 4 ROC curves for the three I/E ratios 


\section{Conclusions}

Our study demonstrated that controlling the $\mathrm{I} / \mathrm{E}$ ratio above the normal physiological level renders a better ability of DC in assessing the sympatho-vagal balance. This is mainly owing to the fact that the elevation of I/E ratio leads to a higher HRA, and thus results in a better quality of phase rectification in the DC calculation.

It is quite demanding for the patients to breathe in an I/E ratio higher than normal for a long time. As a result, the approach may only apply in the short-term DC analysis rather than in the Holter-based standard DC computation. Future researches should focus on studying the mechanisms of HRA to provide better approaches for manipulating the level of HRA.

\section{Acknowledgements}

This study was supported by the Natural Science Foundation of Zhejiang Province (Grant no. LQ14H180001), the National Natural Science Foundation of China (Grant nos.81401491 and 81271662), and Zhejiang Provincial Key Laboratory of Communication Networks and Applications.

No conflicts of interest are declared by the authors.

\section{References}

[1] Bauer A, Kantelhardt JW, Barthel P, et al. Deceleration capacity of heart rate as a predictor of mortality after myocardial infarction: cohort study. Lancet 2006;367(9523):1674-1681.

[2] Bauer A, Kantelhardt JW, Bunde A, et al. Phase-rectified signal averaging detects quasi-periodicities in nonstationary data. Physica A 2006;364:423-434.

[3] Arsenos P and Manis G. Deceleration Capacity of heart rate: Two new methods of computation. Biomed Signal Proces 2014;14(0):158-163.

[4] Bas R, Vallverdú M, Valencia JF, et al. Evaluation of acceleration and deceleration cardiac processes using phase-rectified signal averaging in healthy and idiopathic dilated cardiomyopathy subjects. Med Eng Phys 2015;37(2):195-202.

[5] Pan Q, Zhou G, Wang R, et al. The degree of heart rate asymmetry is crucial for the validity of the deceleration and acceleration capacity indices of heart rate: A modelbased study. Comput Biol Med 2016;76:39-49.

[6] Klintworth A, Ajtay Z, Paljunite A, et al. Heart rate asymmetry follows the inspiration/expiration ratio in healthy volunteers. Physiol Meas 2012;33(10):1717-1731.

[7] Wang YP, Kuo TBJ, and Yang CCH. A possible explanation for the effects of respiration on heart rate and blood pressure asymmetry. Int J Cardiol 2014;174(3):805807.

[8] Ursino M. Interaction between carotid baroregulation and the pulsating heart: a mathematical model. Am J Physiol Heart Circ Physiol 1998;44(5):H1733-H1747.

[9] Ursino $M$ and Magosso E. Role of short-term cardiovascular regulation in heart period variability: a modeling study. Am J Physiol Heart Circ Physiol 2003;284(4):H1479-H1493.

[10] Malpas SC. Sympathetic Nervous System Overactivity and Its Role in the Development of Cardiovascular Disease. Physiol Rev 2010;90(2):513-557.

[11] Porta A, Casali KR, Casali AG, et al. Temporal asymmetries of short-term heart period variability are linked to autonomic regulation. Am J Physiol Regul Integr Comp Physiol 2008;295(2):R550-R557.

Address for correspondence.

Gangmin Ning.

Department of Biomedical Engineering, Zhejiang University, 310027 Hangzhou, China

E-mail address: gmning@zju.edu.cn 